\title{
Obesidade e sobrepeso em trabalhadores da enfermagem de um hospital público em São José dos Campos - SP*
}

\section{Obesity and overweight in nursing workers of a public hospital in São José dos Campos - SP*}

\author{
Renan Sallazar Ferreira Pereira ${ }^{1} \bullet$ Josiane Lima de Gusmão² $\bullet$ Cecília Angelita dos Santos $^{3} \bullet$ Arlete Silva $^{4}$
}

\begin{abstract}
RESUMO
Este estudo descritivo, exploratório, transversal, quantitativo, teve por objetivos caracterizar as variáveis sociodemográficas e analisar o perfil de obesidade e sobrepeso em profissionais de enfermagem. A amostra constituiu-se de 326 trabalhadores de enfermagem de um Hospital geral de São José dos Campos - SP. Observou-se predomínio de mulheres, etnia branca, idade entre 30 e 40 anos, casadas, com renda familiar média de 5 salários mínimos. Quanto aos hábitos, 39,0\% ingeriam bebida alcoólica, 9,9\% eram tabagistas, 78,4\% consumiam alimentos processados e in natura, 38,1\% usavam tempero industrializado e 13,3\% acrescentavam sal nos alimentos depois de pronto. A maioria (66,7\%) era sedentária e estava acima do peso (60\%). Dos 69 (21,1\%) obesos, $79,9 \%$ eram mulheres e $20,3 \%$ homens. A obesidade prevaleceu, entre os homens na faixa de 30 anos, sedentários e que tinham por hábito fumar e beber. Nas mulheres, a obesidade tendeu a aumentar a partir de 40 anos (54,5\%). Destas, 90,9\%, além de obesas, eram sedentárias. É fundamental o acompanhamento multiprofissional para o aconselhamento para hábitos saudáveis, afim de seguirem práticas apropriadas à prevenção e promoção à saúde.
\end{abstract}

Palavras-chave: Enfermagem; Saúde do Trabalhador; Obesidade; Sobrepeso.

\begin{abstract}
This descriptive, exploratory, cross - sectional, quantitative study aimed to characterize sociodemographic variables and analyze the profile of obesity and overweight in nursing professionals. The sample consisted of 326 nursing workers from a general hospital in São José dos Campos - SP. It was observed a predominance of women, white ethnicity, age between 30 and 40 years, married, with average family income of 5 minimum wages. As for the habits, $39.0 \%$ ingested alcoholic beverages, $9.9 \%$ were smokers, $78.4 \%$ consumed processed and in natural foods, $38.1 \%$ used industrialized seasoning and $13.3 \%$ added salt to foods after they are ready. The majority (66.7\%) were sedentary and were overweight (60\%). Of the 69 (21.1\%) obese, $79.9 \%$ were female and $20.3 \%$ male. Obesity prevailed among men in the 30-year age group, who were sedentary and had a habit of smoking and drinking. In women, obesity tended to increase from 40 years (54.5\%). Of these, $90.9 \%$, besides being obese, were sedentary. It is fundamental the multi-professional accompaniment for the advice to healthy habits, in order to follow appropriate practices to the prevention and promotion to the health.
\end{abstract}

Keywords: Nursing; Worker's health; Obesity; Overweight.

\footnotetext{
${ }^{1}$ Recorte do trabalho “Avaliação de Saúde dos Trabalhadores de um Hospital Geral de São José dos Campos - SP”, financiado pela FAPESP na modalidade Projeto Regular (processo n 12/04088-7).

${ }^{2}$ Enfermeiro. Professor Mestre Titular do Centro Universitário UniAges-Paripiranga-BA.

${ }^{3}$ Enfermeira. Professor Adjunto do Programa Mestrado em Enfermagem da Universidade Guarulhos - UnG.

${ }^{4}$ Enfermeira. Hospital Municipal Dr José de Carvalho Florence-São José dos Campos-SP. Enfermeira. Professor Doutor da Universidade de São Paulo (aposentada).

Os autores declaram não haver conflito de interesse.
} 


\section{INTRODUÇÃO}

A obesidade é caracterizada pelo acúmulo excessivo de gordura corporal no indivíduo, resultante da interação de genes, ambiente, estilos de vida, fatores emocionais, metabólicos, comportamentais, sociais e culturais. Pode ser associada com disfunções endócrinas e determinantes ambientais, como exemplo, a diminuição do número de atividades físicas e o abuso da ingestão calórica. A obesidade é diagnosticada quando o IMC alcança valor igual ou superior a $30 \mathrm{~kg} / \mathrm{m}^{2}$, enquanto o excesso de peso/ sobrepeso é diagnosticado com valores de IMC superiores a $25 \mathrm{~kg} / \mathrm{m}^{2}$.

Seu tratamento é complexo, a adesão ao esquema terapêutico é baixa e envolve medicamentos que podem apresentar relação risco-benefício desfavorável ${ }^{2}$. Inibidores do apetite, por exemplo, reduzem o peso durante 0 tratamento, porém o uso prolongado não é recomendado. Medicamentos como a sibutramina podem aumentar o risco de Doenças Cardiovasculares (DCV). Os inibidores da absorção de gordura nos intestinos permanecem como o único medicamento para tratamento de longa duração da obesidade. Como o objetivo do tratamento busca prevenir ou atenuar as morbidades associadas ao sobrepeso e a obesidade, o controle da alimentação em conjunto com a prática de atividades físicas, são hábitos que devem estar associados ao tratamento farmacológico ${ }^{3}$.

Estima-se que em 2025, cerca de 700 milhões de pessoas estarão obesas e mais de 2,3 bilhões de adultos com sobrepeso. Esta pandemia é crescente nos países em desenvolvimento como o Brasil. Dados publicados pelo Ministério da Saúde confirmam o crescimento exponencial da obesidade no Brasil. Em 2014 as pessoas obesas somaram $17,9 \%$ da população e as com sobrepeso ultrapassaram mais da metade da população, cerca de $52,5 \%{ }^{4}$. O crescente aumento da obesidade e a sua associação com comorbidades influem diretamente no bem-estar físico, emocional e psicossocial, com impacto significativo sobre o declínio da qualidade de vida ${ }^{5}$. Além disso, a obesidade prediz o aparecimento das doenças crônicas não transmissíveis (DCNT), que correspondem a $72 \%$ dos óbitos no Brasil. As principais DCNT têm em comum a inatividade física, alimentação não saudável, consumo de bebida alcoólica e tabagismo ${ }^{4}$.

No contexto mundial, em 2012, as DCNT foram responsáveis por 17,5 milhões de mortes. Deste total, 7,5 milhões foram atribuídas a doença arterial coronariana e 6,7 milhões a acidente vascular cerebral ${ }^{6}$. A principal consequência desse quadro é o impacto econômico gerado para o Estado devido ao grande número de internações no Sistema Único de Saúde (SUS), além de antecipar a aposentadoria por doença ou invalidez ${ }^{7}$.

As condições que resultam no fenótipo de obesidade são multicausais e estão enraizados na estrutura do capitalismo, onde o objetivo final é o trabalho humano. 0 ambiente moderno é um potente estímulo para a obesidade $^{8}$. Empresas que exigem alta produtividade geram estresse psicológico, ansiedade e depressão e, consequentemente, podem fazer com que os trabalhadores criem o hábito de se alimentar rapidamente, procurando fast foods e reduzindo o tempo para a prática de atividades físicas, contribuindo assim para o aumento dos indicadores de obesidade?

No Brasil, um estudo demonstrou que os trabalhadores de enfermagem possuem risco aumentado para DCV, principalmente aqueles que executam atividades assistenciais. Dentre os fatores de risco encontrados neste contingente, destacam-se a obesidade, circunferência abdominal alterada, hipertensão, história familiar e estresse ${ }^{10}$.

Conhecer as condições de saúde do trabalhador e promover saúde por meio de adoção de hábitos saudáveis beneficia os trabalhadores de enfermagem e possibilita a prestação de assistência de melhor qualidade ${ }^{11}$.

Este estudo teve como objetivo caracterizar as variáveis sociodemográficas e analisar o perfil da obesidade e sobrepeso em trabalhadores da enfermagem de um hospital geral, público de São José dos Campos-SP.

\section{MÉTODO}

Trata-se de um estudo descritivo, transversal, de campo, com abordagem quantitativa, realizado com 326 profissionais de enfermagem, sendo 66 (20,2\%) enfermeiros, 177 (54,3\%) técnicos de enfermagem e 83 (25,5\%) auxiliares de enfermagem; é um recorte do estudo primário denominado "Avaliação de Saúde dos Trabalhadores de um Hospital Geral de São José dos Campos - SP", que teve por objetivo geral avaliar as condições de saúde dos profissionais que trabalham nesse hospital e que foi aprovado pelo Comitê de Ética em Pesquisa da Universidade de Taubaté (UNITAU) sob Parecer n 556/11.

Para a obtenção da amostra, considerou-se a prevalência de hipertensão de $30 \%$ nos trabalhadores de enfermagem do hospital, sendo o erro de $5 \%$ e intervalo de confiança de $95 \%$.

O hospital campo de estudo foi inaugurado em 1978, conta com 307 leitos e 1.795 trabalhadores de todas as categorias profissionais. É vinculado à Secretaria Municipal de Saúde de São José dos Campos e gerenciado pela Associação Paulista Para o Desenvolvimento da Medicina (SPDM) desde 2006; reconhecido como Hospital Amigo da Criança, recebeu o Prêmio COREN-SP de "Gestão com Qualidade Dimensão Hospitalar Edição 2011/2012.

A coleta de dados foi feita por meio de questionários e as variáveis de interesse selecionadas para o presente estudo foram: dados pessoais (data de nascimento, sexo, etnia, estado civil, nacionalidade, cidade onde mora); dados profissionais (tempo de serviço na instituição, local de trabalho, ocupação/ cargo, renda familiar, horas diárias de trabalho na instituição, outras atividades e trabalho 
aos fins de semana); estilo de vida (ingestão de bebida alcoólica, tabagismo, prática de exercício físico e hábitos alimentares); e de exame físico (índice de massa corporal IMC e circunferência abdominal).

O IMC foi calculado pela fórmula "peso dividido por estatura ao quadrado", observando as medidas do peso em quilogramas e a da estatura em metros. As medições foram realizadas em balança digital com antropômetro, marca Filizola, modelo PL180. Na ocasião, os indivíduos ficaram com roupas leves e retiraram os sapatos. Para avaliação da estatura foi solicitado que o indivíduo ficasse ereto na posição ortostática, com os braços ao longo do corpo, os pés unidos e a cabeça erguida com olhos fixos no horizonte. 0 IMC foi classificado como. Baixo peso $\left(<18,4 \mathrm{~kg} / \mathrm{m}^{2}\right)$, Peso normal $\left(18,5-24,9 \mathrm{~kg} / \mathrm{m}^{2}\right)$; Sobrepeso $\left(25,0-29,9 \mathrm{~kg} / \mathrm{m}^{2}\right)$; Obeso $\left(\geqslant 30,0 \mathrm{~kg} / \mathrm{m}^{2}\right)^{12}$.

A circunferência abdominal foi medida por meio de fita milimetrada flexível, não distensível, no ponto médio entre o rebordo costal inferior e a crista ilíaca. Foram considerados valores elevados aqueles superiores a 102 $\mathrm{cm}$ para homens e $88 \mathrm{~cm}$ para mulheres.

Foram considerados sedentários os indivíduos que realizam menos que 30 minutos/dia de atividade física por duas, uma ou nenhuma vez na semana ${ }^{12}$.

Considerou-se tabagista aquele indivíduo que consome regularmente, no mínimo, um cigarro por dia, pelo menos durante um ano ou que tenha fumado no ano anterior a participação neste estudo. Ex-fumantes aqueles que não fumaram no ano anterior a inclusão neste estudo, mas que o tenham feito entre um e 10 anos anteriores à pesquisa. Não fumantes aqueles que nunca haviam fumado ou o haviam feito há mais de 10 anos $^{12}$.

Os dados foram armazenados em banco de dados criado em planilha no programa de computador Excel, analisados quantitativamente e apresentados em forma de tabelas. Foi realizada análise descritiva que utilizou medidas de tendência central (média, máxima e mínima) e medida de dispersão (desvio padrão) para as variáveis contínuas e número absoluto e frequência relativa para as variáveis categóricas.

\section{RESULTADOS}

Ao considerar todas categorias, observa-se que a maioria da amostra estudada é composta por mulheres ( $n=260 ; 84,38 \%)$, brancas ( $n=229 ; 72,7 \%)$, na faixa etária de 30 a 40 anos ( $n=147 ; 46,0 \%$ ), sendo a idade mínima 24 e a máxima 58 anos, com média de $34,6(\mathrm{dp} \pm 8,2)$ e casados/ amasiados ( $\mathrm{n}=172 ; 52,9 \%)$.

Em relação às características profissionais, a média de tempo de atuação dos trabalhadores no hospital foi de 4,1(dp $\pm 3,7)$ anos, sendo o tempo mínimo de 1 ano e o máximo de 25 anos; apenas $1(0,3 \%)$ trabalhador atuava no hospital há mais de 24 anos.

Quanto ao local de lotação, exatamente 61 (21,2\%) estão no pronto socorro adulto, $47(16,4 \%)$ na clínica médica e 63 (21,9\%) na UTI adulto e UTI infantil e 29 $(10,1 \%)$ na maternidade e pediatria, 12 (4,2\%) na central de materiais e esterilização. Foram referidas ainda o ambulatório médico e atendimento pré-hospitalar, com igual percentual (8; 2,8\%).

Considerando todas as categorias de enfermagem, a maioria (202; 65,6\%) declarou renda familiar entre 3 a 7 salários mínimos, observando-se maior renda entre os enfermeiros (2,5 a 16,7 salários mínimos).Aproximadamente um terço $(n=105 ; 33,2 \%)$ dos trabalhadores exerciam outra atividade profissional em outra instituição, sendo a maioria deles ( $n=75 ; 78,8 \%)$ na área da saúde, seguida da educação ( $n=13 ; 13,6 \%)$. A maioria $(n=251 ; 87,5 \%)$ trabalha aos finais de semana em regime de plantão de $12 \times 36 \mathrm{~h}$. Quanto aos hábitos e estilo de vida, 126 (39,0\%) referiram ingerir bebida alcoólica, 32 (9,9\%) fumar e 210 (78,8\%) foram classificados como sedentários (tabela 1).

A maioria $(254 ; 78,4 \%)$ declarou consumir alimentos processados e in natura, 122 (38,1\%) usam tempero industrializado e $43(13,3 \%)$ acrescentam sal nos alimentos depois de pronto. Nos dados apresentados na tabela 2, nota-se que o método de preparo mais utilizado foi o cozido (197; 60,4\%).

Pelos dados da tabela 3, verifica-se que 127 (39,0\%) trabalhadores estavam com o peso normal, 126 (38,7\%) com sobrepeso e 69 (21,1\%) com obesidade, o que significa que quase $60 \%$ da amostra estavam acima do peso. A maioria dos enfermeiros apresentou peso normal (34; 51,6\%). Com as demais categorias ocorreu o oposto, 64 (36,2\%) técnicos e 29 (34,9\%) somente apresentaram peso normal.

A prevalência da obesidade foi de 14 (20,3\%) no sexo masculino e 55 (79,9\%) no feminino.

Ao considerar a relação da prevalência da obesidade com alguns dados sociodemográficos (sexo, idade, renda), hábitos inadequados (etilismo, tabagismo e sedentarismo), verifica-se a prevalência em homens a partir dos 30 anos, com média de renda de 5 salários mínimos, não fumantes (9; 64,3\%), consumidores de bebidas alcoólicas (8; 57,1\%) e sedentários (11; 78,6\%). Nas mulheres, a obesidade tendeu a aumentar a partir de 40 anos (54,5\%), destas, (90,9\%), além de obesas eram sedentárias. Observa-se ainda que a maioria dos trabalhadores $(240 ; 73,6 \%)$ apresentavam medida anormal de circunferência abdominal.

\section{DISCUSSÃO}

A presença de fatores de risco, como dieta inadequada, obesidade, sedentarismo, circunferência abdominal elevada, estresse está relacionada às características do trabalho da enfermagem, inclusive o excesso de trabalho ${ }^{13}$.

Fatores sociodemográficos e comportamentais estão associados ao aumento do peso corporal. Estudo realizado nas 27 capitais brasileiras apontou prevalência da obesidade de $18 \%$ na população de adultos, sem diferença entre os sexos. Em ambos os sexos, a frequência 
Tabela 1: Distribuição dos trabalhadores de enfermagem, segundo hábitos e estilo de vida. São José dos Campos, SP, Brasil, 2015.

\begin{tabular}{|c|c|c|c|c|c|c|c|c|}
\hline \multirow{2}{*}{ Variáveis } & \multicolumn{2}{|c|}{ Enfermeiro } & \multicolumn{2}{|c|}{ Técnico de enf. } & \multicolumn{2}{|c|}{ Auxiliar de enf. } & \multicolumn{2}{|c|}{ Total } \\
\hline & $\mathbf{n}$ & $\%$ & $\mathbf{n}$ & $\%$ & $\mathbf{n}$ & $\%$ & $n$ & $\%$ \\
\hline \multicolumn{9}{|l|}{ Etilista } \\
\hline Sim & 31 & 47,0 & 66 & 37,5 & 29 & 35,8 & 126 & 39,0 \\
\hline Não & 35 & 53,0 & 110 & 62,5 & 52 & 64,2 & 197 & 61,0 \\
\hline Total* & 66 & 100,0 & 176 & 100,0 & 81 & 100,0 & 323 & 100,0 \\
\hline \multicolumn{9}{|l|}{ Tabagista } \\
\hline Sim & 7 & 10,6 & 12 & 6,9 & 13 & 16,00 & 32 & 9,9 \\
\hline Não & 59 & 89,4 & 163 & 93,1 & 68 & 84,0 & 290 & 90,1 \\
\hline Total* & 66 & 100,0 & 175 & 100,0 & 81 & 100,0 & 322 & 100,0 \\
\hline \multicolumn{9}{|l|}{ Sedentário } \\
\hline Sim & 41 & 65,0 & 147 & 84,5 & 64 & 77,1 & 210 & 65,6 \\
\hline Não & 22 & 35,0 & 27 & 15,5 & 19 & 22,9 & 110 & 34,4 \\
\hline Total $^{*}$ & 63 & 100,0 & 174 & 100,0 & 83 & 100,0 & 320 & 100,0 \\
\hline
\end{tabular}

${ }^{*} n \neq 326$ : alguns participantes da pesquisa não responderam estas variáveis.

Fonte: Elaborado pelos próprios autores.

Tabela 2: Distribuição dos trabalhadores de enfermagem, segundo o preparo dos alimentos. São José dos Campos, SP, Brasil, 2015.

\begin{tabular}{|c|c|c|c|c|c|c|c|c|c|}
\hline \multirow{2}{*}{ Categoria } & \multicolumn{2}{|c|}{ Cozido } & \multicolumn{2}{|c|}{ Assado } & \multicolumn{2}{c|}{ Grelhado } & \multicolumn{2}{c|}{ Frito } & \multicolumn{2}{c|}{ Ensopado } \\
\cline { 2 - 10 } & $\mathbf{n}$ & $\%$ & $\mathbf{n}$ & $\%$ & $\mathbf{n}$ & $\%$ & $\mathbf{n}$ & $\%$ & $\mathbf{n}$ \\
\hline
\end{tabular}

\section{Enfermeiro}

\begin{tabular}{l|r|r|r|r|r|r|r|r|rr}
\hline Sim & 33 & 50,0 & 15 & 22,7 & 13 & 19,7 & 16 & 24,2 & 3 & 4,6 \\
\hline Não & 33 & 50,0 & 51 & 77,3 & 53 & 80,3 & 50 & 75,8 & 63 & 95,4 \\
\hline Total & 66 & 100,0 & 66 & 100,0 & 66 & 100,0 & 66 & 100,0 & 66 & 100,0 \\
\hline
\end{tabular}

Técnico de enfermagem

\begin{tabular}{l|c|r|r|r|r|r|l|r|rr}
\hline Sim & 112 & 63,3 & 58 & 32,8 & 34 & 19,2 & 55 & 31,1 & 23 & 13,0 \\
\hline Não & 65 & 36,7 & 119 & 67,2 & 143 & 80,8 & 122 & 68,9 & 154 & 87,0 \\
\hline Total & 177 & $\mathbf{1 0 0 , 0}$ & $\mathbf{1 7 7}$ & $\mathbf{1 0 0 , 0}$ & $\mathbf{1 7 7}$ & $\mathbf{1 0 0 , 0}$ & $\mathbf{1 7 7}$ & $\mathbf{1 0 0 , 0}$ & $\mathbf{1 7 7}$ & $\mathbf{1 0 0 , 0}$ \\
\hline
\end{tabular}

Auxiliar de enfermagem

\begin{tabular}{l|r|r|r|r|r|r|r|r|rr}
\hline $\operatorname{Sim}$ & 31 & 37,3 & 26 & 31,3 & 17 & 20,4 & 30 & 36,1 & 14 & 16,9 \\
\hline Não & 52 & 62,7 & 57 & 68,7 & 66 & 79,6 & 53 & 63,9 & 69 & 83,1 \\
\hline Total & $\mathbf{8 3}$ & $\mathbf{1 0 0 , 0}$ & $\mathbf{8 3}$ & $\mathbf{1 0 0 , 0}$ & $\mathbf{8 3}$ & $\mathbf{1 0 0 , 0}$ & $\mathbf{8 3}$ & $\mathbf{1 0 0 , 0}$ & $\mathbf{8 3}$ & $\mathbf{1 0 0 , 0}$ \\
\hline
\end{tabular}

Enfermeiro/Técnico/ Auxiliar

\begin{tabular}{l|r|r|r|r|r|r|r|r|rr}
\hline $\operatorname{Sim}$ & 197 & 60,4 & 99 & 30,4 & 64 & 19,6 & 101 & 31,0 & 40 & 12,3 \\
\hline Não & 129 & 39,6 & 227 & 69,6 & 262 & 80,4 & 225 & 69,0 & 286 & 87,7 \\
\hline Total & 326 & 100,0 & 326 & 100,0 & 326 & 100,0 & 326 & 100,0 & 326 & 100,0 \\
\hline
\end{tabular}

Fonte: Elaborado pelos próprios autores.

Tabela 3: Distribuição dos trabalhadores de enfermagem, segundo o Índice de Massa Corporal (IMC) e medida da circunferência abdominal. São José dos Campos, SP, Brasil, 2015.

\begin{tabular}{|c|c|c|c|c|c|c|c|c|}
\hline \multirow{2}{*}{ Variáveis } & \multicolumn{2}{|c|}{ Enfermeiro } & \multicolumn{2}{|c|}{ Técnico de enf. } & \multicolumn{2}{|c|}{ Auxiliar de enf. } & \multicolumn{2}{|c|}{ Total } \\
\hline & $\mathrm{n}$ & $\%$ & $\mathrm{n}$ & $\%$ & $\mathbf{n}$ & $\%$ & $\mathrm{n}$ & $\%$ \\
\hline \multicolumn{9}{|l|}{ IMC } \\
\hline Baixo peso & 2 & 3 & 2 & 1,1 & 0 & 0,0 & 4 & 1,2 \\
\hline Peso normal & 34 & 51,6 & 64 & 36,2 & 29 & 34,9 & 127 & 39,0 \\
\hline Sobrepeso & 17 & 25,8 & 70 & 39,6 & 39 & 47,0 & 126 & 38,7 \\
\hline Obeso & 13 & 19,6 & 41 & 23,1 & 15 & 18,1 & 69 & 21,1 \\
\hline Total & 66 & 100,0 & 177 & 100,0 & 83 & 100,0 & 326 & 100,0 \\
\hline \multicolumn{9}{|c|}{ Circunferência abdominal } \\
\hline Normal & 26 & 39,4 & 37 & 20,9 & 23 & 27,7 & 86 & 26,4 \\
\hline Anormal & 40 & 60,6 & 140 & 79,1 & 60 & 72,3 & 240 & 73,6 \\
\hline Total & 66 & 100,0 & 177 & 100,0 & 83 & 100,0 & 326 & 100,0 \\
\hline
\end{tabular}

Fonte: Elaborado pelos próprios autores. 
da obesidade tendeu a aumentar com a idade até os 44 $a_{n o s}{ }^{4}$, ou seja, a média nacional é mais tardia do que no presente trabalho.

A preferência por alimentos cozidos foi positiva. No entanto, preocupa o grande número de trabalhadores optando por alimentos industrializados. A alta concentração de sódio e outros conservantes podem contribuir para o aumento de fatores de risco cardiovascular ${ }^{13}$.

O IMC está associado com o risco de morte. Estudo realizado com 1124897 participantes mostrou que homens e mulheres com índice de massa corporal igual ou superior a $25,0 \mathrm{Kg} / \mathrm{m}^{2}$, tiveram um risco aumentado de morte por doenças cardiovasculares, incluindo doenças cardíaca coronariana, acidente vascular cerebral isquêmico e hemorrágico ${ }^{14}$. No presente estudo, aproximadamente $60 \%$ apresentaram IMC acima do recomendado.

O sobrepeso e a obesidade estão associados ao aumento da morbimortalidade, pois favorece a ocorrência de co-morbidades, tais como aumento do risco para as dislipidemias, hipertensão, diabetes, síndrome metabólica. Além disso, a obesidade leva a complicações cardiovasculares, contribui para a hipertrofia cardíaca, alteração da parede ventricular esquerda, disfunção diastólica e acúmulo de lipídios no coração devido à desregulação entre a captação de ácidos graxos e oxidação, entre outros problemas ${ }^{14-15}$.

Os dados levantados pela VIGITEL ${ }^{4}$ em 2014 nas 26 capitais e no Distrito Federal mostrou que a frequência do excesso de peso $\left(25,0-29,9 \mathrm{Kg} / \mathrm{m}^{2}\right)$ foi de $52,5 \%$, sendo maior entre homens $(56,5 \%)$ do que entre mulheres (49,1\%). Os achados do presente estudo mostraram um comportamento diferente, houve 3 vezes mais de mulheres obesas $(55 ; 79,9 \%)$ do que homens $(14 ; 20,3 \%)$.

No presente estudo, o número de trabalhadores obesos chegou a 69 (21,1\%), ou seja, superou a média nacional (17,9\%) de obesos apresentado pela VIGITEL ${ }^{4}$.Vale ressaltar que a obesidade nos trabalhadores da enfermagem tem início mais cedo, tende a aumentar a partir dos 30 anos nos homens e 40 anos nas mulheres. Isto mostra a gravidade da obesidade nos trabalhadores de enfermagem do hospital estudado e indica a necessidade de reduzirem o peso para minimizar o risco cardiovascular e prevenir a DM do tipo 2 entre outras complicações causadas pela obesidade.

Considerando as três categorias profissionais, observase que a maior prevalência de sobrepeso/obesidade foi entre os auxiliares de enfermagem (54; 65,1\%) e técnicos (111; 62,7\%), comparado com os enfermeiros (30; (45,4\%). Este dado já era previsto, pois a frequência da obesidade e do sobrepeso tende a diminuir com o aumento do nível de escolaridade 4 .

Em relação à circunferência abdominal, verifica-se que 240 (73,6\%) apresentavam circunferência abdominal elevada, ou seja, mais de $70 \%$ da amostra apresentavam risco para complicações metabólicas. A medida da circunferência abdominal reflete o conteúdo de gordura visceral e também se associa à gordura corporal total, sendo, portanto, um bom preditor de risco para doenças metabólicas e da hipertensão arterial sistêmica ${ }^{16}$.

Em relação ao acúmulo de gordura na região abdominal, ela é um fator de risco cardiovascular que predispõe à diabete melito do tipo 2 e síndrome metabólica, aumentando o risco cardiovascular, hiperglicemia; hipertrigliceridemia, apolipoproteína B e lipoproteína de baixa densidade (LDL); e diminuição na lipoproteína de alta densidade (HDL). 0 excesso de gordura abdominal apresenta maior risco cardiovascular do que o excesso de gordura corporal por si só ${ }^{17}$.

A obesidade abdominal é considerada fator de risco independente para o desenvolvimento de doenças cardiovasculares, principalmente a diabetes tipo $\mathrm{II}^{18}$. Os resultados obtidos no presente estudo mostrou que, a maioria (240; 73,6\%) apresentou obesidade abdominal, portanto, apresentam risco cardiovascular.

\section{CONCLUSÃO}

A prevalência de sobrepeso e obesidade entre os trabalhadores de enfermagem foi elevada no presente estudo e superior à média nacional. Esse achado causa bastante preocupação pelo fato de sugerir que a sobrecarga de trabalho deixa pouco tempo para os trabalhadores de enfermagem promoverem o autocuidado, principalmente no diz respeito à dificuldade de adotar um estilo de vida mais saudável. Com isto, aumenta o sedentarismo, propicia a dieta inadequada, entre outros problemas que levam à obesidade e aos demais fatores de risco cardiovascular.

Os trabalhadores de enfermagem necessitam de acompanhamento multiprofissional para desenvolverem hábitos saudáveis, a fim de seguirem técnicas apropriadas para aderirem à prática preventiva e de promoção à saúde, como a prática regular de atividade física e dieta equilibrada. Soma-se a isto a necessidade de promover a melhoria das condições de trabalho na instituição de saúde.

\section{REFERÊNCIA}

1 Associação Brasileira para o estudo da obesidade e da síndrome metabólica [Internet]. Brasil; 2015. [acesso 16 nov. 2016] Disponível em: http://www.abeso.org.br/atitudesaudavel/mapa-obesidade.

2 Yanovski SZ, Yanovski JA. Long-term Drug Treatment for Obesity. A Systematic and Clinical Review. JAMA [Internet]. 2014 [acesso em 02 mai 2017]; 311(1):74-86. Disponível em: https://www.ncbi.nlm.nih.gov/pubmed/24231879.

3 Ryan DH, Bray GA. Pharmacologic treatment options for obesity: what is old is new again. Curr Hypertens Rep [Internet]. 2013 [acesso em 02 mai 2017]; 15(3):182-189. Disponível em: https://www.ncbi.nlm.nih.gov/pubmed/23625271.

4 Brasil. Ministério da Saúde. Secretaria de Vigilância em Saúde. Departamento de Vigilância de Doenças e Agravos não Transmissíveis e Promoção da Saúde. Brasília: Ministério da Saúde [Internet]. 2015 [acesso 16 dez. 2016] Disponível 
em. http://bvsms.saude.gov.br/bvs/publicacoes/vigitel_ brasil_2014.pdf.

5 Cameron AJ, Magliano DJ, Dunstan DW, Zimmet PZ, et al. A bi-directional relationship between obesity and healthrelated quality of life: evidence from the longitudinal AusDiab study, International journal of obesity. [Internet]. 2012 [acesso em 15 mai 2017]; 36(2): 295-303. Disponível em: https://dro.deakin.edu.au/eserv/DU:30046272/cameronbidirectionalrelationship-2012.pdf.

6 World Health Organization. Global status report on no communicable diseases 2014 [Internet]. Switzerland; [acesso 10 mai. 2017] Disponível em: http://apps.who.int/iris/ bitstream/10665/148114/1/9789241564854_eng.pdf?ua=1.

7 Bahia LR, Araújo DV. Impacto econômico da obesidade no Brasil. Revista HUPE [Internet]. 2014 [acesso em 05 mai 2017]; (1):13-17. Disponível em: http://www.e-publicacoes. uerj.br/index.php/revistahupe/article/view/9793/8763.

8 Egger G, Dixon J. Beyond obesity and lifestyle: a review of 21st century chronic disease determinants. Biomed Res Int [Internet]. 2014 [acesso em 04 mai 2017]; 14(12): 2-8. Disponível em: https://www.hindawi.com/journals/ bmri/2014/731685/.

9 Ramin C, Devore EE, Wang W, Wang W, et al. Night shift work at specific age ranges and chronic disease risk factors Occup Environ Med [Internet]. 2015 [acesso em 05 mai 2017]; 72:100-107. Disponível em: https://www.ncbi.nlm.nih.gov/ pmc/articles/PMC4289641/.

10 Magalhães FJ, Mendonças LBA, Rebouças CBA, Lima FET, et al. Fatores de risco para doenças cardiovasculares em profissionais de enfermagem: estratégias de promoção da saúde. Rev Bras Enferm [Internet]. 2014 [acesso em 05 mai 2017]; 67(3):394-400. Disponível em: http://www.scielo.br/ pdf/reben/v67n3/0034-7167-reben-67-03-0394.pdf.

11 Letvak, SA, Ruhm, CJ, Gupta S N. Original research: Nurses' presentism and its effects on self-reported quality of care and costs. AJN The American Journal of Nursing [Internet]. 2012 [acesso; 112(2): 30-38. Disponível em: https://www.ncbi.nlm. nih.gov/pubmed/22261652.

12 Jensen MD, Ryan DH, Apovian CM, Ard JD, et al. . 2013 AHA/ACC/ TOS guideline for the management of overweight and obesity in adults: a report of the American College of Cardiology/ American Heart Association Task Force on Practice Guidelines and The Obesity Society. Circulation [Internet]. 2013 [acesso em 05 nov 2015] 129(25): 102-38. Disponível em: http://circ.ahajournals.org/content/suppl/2013/11/07/01. cir.0000437739.71477.ee.DC1.

13 Schwingshackl L, Hoffmann G. Diet quality as assessed by the Healthy Eating Index, the Alternate Healthy Eating Index, the Dietary Approaches to Stop Hypertension score, and health outcomes: A systematic review and meta-analysis of cohort studies. J Acad Nutr Diet [Internet]. 2015 [acesso em 07 mai 2017]; 115:780-800. Disponível em: https://www.ncbi.nlm. nih.gov/pubmed/25680825.

14 O'Brien PD, Hinder LM, Callaghan BC, Feldman EL. Neurological consequences of obesity.Lancet Neurol [Internet]. 2017 [acesso em 08 mai 2017]; 16(6):465-477. Disponível em: http://www. sciencedirect.com/science/article/pii/S1474442217300844.

15 Macedo TTS, Palmeira CS, Guimarães AC, Lima ML, et al. 0 significado da obesidade: a precepção do paciente obeso. J
Nurs UFPE on line [Internet]. 2013 [acesso em 16 mai 2017]; 7 (12): 7064-73. Disponível em: http://www.revista.ufpe.br/ revistaenfermagem/index.php/revista/article/view/4714/ pdf_4257.

16 Carvalho CA, Fonseca PCA, Barbosa JB, Machado SP, et al. Associação entre fatores de risco cardiovascular e indicadores antropométricos de obesidade em universitários de São Luís, Maranhão, Brasil. Ciência \& Saúde Coletiva [Internet]. 2015 [acesso em 11 mai 2017]; 20 (2): 490-90. Disponível em: http:// www.scielo.br/pdf/csc/v20n2/1413-8123-csc-20-02-0479.pdf.

17 Pavanelli MF, Arantes VC, Ribas-Silva RC. Relação entre adiposidade visceral e hipertensão arterial sistêmica. Rev Enferm UFSM [Internet]. 2012 [acesso em 11 mai 2017]; 2(3):584-90. Disponível em: file:///C:/Users/renansallazar/ Downloads/6326-34851-1-PB.pdf.

18 Marinho NBP, Vaconcelos HCA, Alencar AMPG, Almeida PC, et al. Risco para diabetes mellitus tipo 2 e fatores associados. Acta Paul Enferm [Internet]. 2013; 26(6):569-74. Disponível em: http://www.scielo.br/scielo.php?script=sci_ arttext \& pid=S0103-21002013000600010. 